\title{
Uncertainty in labor productivity and specific human capital investment
}

\section{Uncertainty in Labor Productivity and Specific Human Capital Investment}

\author{
Chong-En Bai, University of Hong Kong \\ Yijiang Wang, University of Minnesota
}

\begin{abstract}
Uncertainty in labor productivity (ULP) is affected by many factors, such as worker-employcr matching, technology, and macroeconomic conditions. Not surprisingly, ULP varies across firms, industries, and economies. How do variations in ULP affect specific human capital (SHC) investment, wage, and labor turnover? A fixed-wage model is used to show that the answer depends critically on the initial level of ULP. The model is also used to show that wage and SHC are always positively correlated, but SHC investment and labor turnover do not have a monotonic relationship. These results have implications for empirical studies and public policies affecting ULP.
\end{abstract}

\section{Introduction}

Since Becker's (1975) seminal work, the importance of firm-specific human capital (SHC) to a worker's productivity has received much at-

We thank first and foremost Eric Maskin for his invaluable advice. We also thank Morris Kleiner, Stephanie Lluis, Brian McCall, and David Scoones for their many constructive comments and many specific suggestions, as well as seminar participants at Harvard University, Boston College, Boston University, London School of Economics, University of Minnesota, Stanford University, and University of Wisconsin-Madison for helpful comments. The financial support from Hong Kong RGC Grant HKU7135/00H (Bai), University of Minnesota's Grantin-Aid of Research, Artistry and Scholarship (Wang, no. 1043-331-5981), and McKnight Summer Research Grant (Wang, 2000 and 2001) are gratefully acknowledged.

[Joumal of Labor Economics, 2003, vol. 21, no. 3]

(C) 2003 by The University of Chicago. All rights reserved.

0734-306X/2003/2103-0006\$10.00 
tention. 'Meanwhile, it is well understood that productivity is also affected by factors such as uncertainty in job matching quality. The degree of uncertainty in labor productivity (ULP) can vary across firms for different reasons. Miller (1984) and McCall (1994), for instance, noticed that larger firms may face less ULP because more internal job choices can help to improve the worker (he)-employer (she) match. Also, firms in industries characterized by new technology, new products, or greater competition may face more ULP than firms in industries typified by relatively mature technology, mature products, and protected markets. Still another possible reason for differences in ULP is international differences in macroeconomic stability. ${ }^{2}$

While ULP arguably influences SHC investment, ${ }^{3}$ little is known about how this operates. Since ULP is evident at the macro, industry, and firm levels, its influence on investment may translate into important effects on, for example, wages and employment stability. ${ }^{+}$The following questions arise: As a worker's productivity becomes less predictable, will he invest more or less in SHC? Can he expect a higher or lower wage? Is he more or less likely to separate from the current employer? Answers to these

\footnotetext{
'Important contributions to human capital theory have also been made by Mincer (1962), Oi (1962), Parsons (1972), Rosen (1972), Mortensen (1978), Jovanovic (1979a), Kennan (1979), Hashimoto and Yu (1980), Hashimoto (1981), Carmichacl (1983), Bcrnhardt and Timmis (1990), Prendergast (1993), Scoones and Bernhardt (1998), Scoones (2000), and others. Waldman (1990) and Chang and Wang (1996) argued that general human capital can be viewed as firm specific in the "as if" sense for informational reasons.

${ }^{2}$ Many economic historians have observed the high variability of growth at the early stages of economic development (see North and Thomas [1973]; McCloskey [1976]; Braudel [1979]; and DeVries [1990]; and Accmoglu and Zilibotti [1997], for some examples). Acemoglu and Zilibotti (1997) explain this greater uncertainty in developing economies by limited opportunities for investors to diversify idiosyncratic risks. Comparing two industrialized cconomies, Taylor (1989) finds that from 1976 to 1986 , the standard deviation of the percentage quarterly real GNP fluctuations around a constant exponential trend is $2.5 \%$ for the United States and $1.1 \%$ for Japan. This difference is significant at the $99 \%$ level. This means that an "average" Japanese firm faces less ULP than its American counterpart. Bai and Wang (1996) calculated that, from 1973 to 1987, the standard deviations of output (in terms of value added) from the trend values are higher in the United States than in Japan in five of the cight manufacturing industries, four of them at the $99 \%$ level and one at the $95 \%$ level. These five industries in 1985 produced $83 \%$ of the total value added by the manufacturing sector. In the other three industries, the differences in output fluctuations are not statistically significant.

${ }^{3}$ This is the essence of the works by Hashimoto (1981) and Parsons (1972). See the review by Parsons (1986) and that by Gibbons and Waldman (1999).

'There is a literature on firms' investment decisions under uncertainty. It focuses on the option value of delaying investment and waiting for new information in a dynamic environment. For a review, see Dixit and Pindyck (1994). Our model is very different from this literature.
} 
questions can help us to better address policy questions such as, When a particular industry becomes more or less volatile due to changes in technological or market conditions, what can we expect to happen to wage and employment stability in that industry? When we enact policies with an effect on ULP to intervene in the market, what are we doing to productivity, wage, and employment stability?

In this article, we present a fixed wage contract model to study the effects of ULP on the three key employment variables mentioned above: SHC investment, wage, and the probability of separation. ${ }^{6}$ We find that neither the firm's wage offer to nor the amount of SHC investment by the worker changes monotonically with ULP, that is, greater ULP sometimes leads to a lower wage offer and less SHC investment, but other times the opposite. Greater ULP also sometimes increases but other times reduces the probability of separation. Wage and $\mathrm{SHC}$ are always positively correlated, but SHC investment and the probability of separation do not have a monotonic relationship. Depending on parameter values, more SHC investment can be either positively or negatively associated with the probability of separation.?

The reasoning for these results is as follows. In a fixed wage contract, the employer offers the worker a wage above his market wage. Then the worker has an incentive to invest in SHC to reduce the probability of separation. The marginal effectiveness of SHC investment in reducing the probability of separation is determined by the density of the productivity distribution at the offered wage. As we will show, when ULP is very small, the wage will fall into the lower tail of the productivity distribution. When greater ULP implies a "fatter" lower tail (as true for many distributions), it also means that, at the original equilibrium wage, SHC investment is marginally more effective in reducing the probability of separation. Thus, the worker finds it worthwhile to invest more in SHC. The improved effectiveness of wage in inducing SHC investment also

${ }^{5}$ Many countries have macro stabilization fiscal and monetary policies. In Japan, additional institutional factors contribute to less volatility in the economy and industries, e.g., government planning (Johnson 1988), the main bank system (Horiuchi, Packer, and Fukuda 1988; A oki 1990), informal school-cmployer linkages (Rosenbaum and Kariya 1989), and multiskilling (Carmichacl and MacLeod 1993).

'Since Keynes (1936), wage rigidity (the lack of the ability for employers to adjust wage downward at times of low labor productivity) has been widely recognized. Keynes and his followers see this as a major explanation for layoffs and uncmployment. Shapiro and Stiglitz (1986), Kahn and Huberman (1988), and Waldman (1990) offer cxplanations of the phenomenon based on various labor market incentive and informational problems, which we will further explain later.

${ }^{7}$ Scoones (2000) also obtains the result that the probability of turnover and worker investment in SHC may be positively correlated. However, both the model and the underlying logic of the result are quite different from those offered here. His is a matching model with employer competition and efficient turnover. 
leads the employer to offer a higher wage. ${ }^{8}$ The opposite is true when ULP is high because then the equilibrium wage will be close to the mean of the probability distribution where the density becomes lower as ULP increases.

While very high ULP leads to very low SHC investment, wage is bounded from below by the worker's market wage. This means that, when ULP is very high, the expected productivity can be so low as to fall below the wage. When this is true, the likelihood that realized productivity is above the wage actually increases with ULP, meaning a smaller probability of separation when ULP becomes greater." When this direct, negative effect of greater ULP on separation dominates its indirect, positive effect through the downward adjustment in SHC investment, the probability of separation is smaller. We thus see SHC investment and the probability of separation move in the same direction, that is, they both decrease when ULP is greater.

The plan for the rest of the article is as follows. Section II presents the model. Section III characterizes the equilibrium. Section IV studies the comparative statics of wage, SHC investment, and separation with respect to changes in ULP. Section V further analyzes the effect of ULP on separation, focusing on a particular specification of the measure of ULP. Section VI discusses empirical implications of the model. Section VII concludes.

\section{The Model}

In a game between the employer and the worker, wage, the level of the worker's SHC investment, and the probability of separation are endogenously determined. The game also has the following specifics.

The events and their sequence.-First, the employer offers an employment contract to the worker. The worker accepts or rejects it. If the worker rejects the offer, the game ends. If the worker accepts it, he proceeds to make an investment in SHC before production starts. The level of the $\mathrm{SHC}$ investment is the worker's private information. After the investment, the worker's productivity is revealed to the employer but not the worker. The employer and the worker then decide if the employment relationship

${ }^{8}$ This discussion suggests that, if monopoly or regulation (its extreme form being ccntral planning) leads to very small ULP in an industry, antitrust, deregulation, or market-oriented reform that subjects firms to more competition and thereby greater ULP can improve efficiency in "effort" besides that in pricing behavior of the firms. The "complacency" problem suggested by Hicks (1935) and commonly observed in monopolistic and regulated industrics can be seen in the light of this discussion.

"Some people prefer to work for smaller firms with less pay and less job security for better promotion and other opportunities. Higher ULP may be interpreted as greater "vertical mobility" in this kind of firm. 


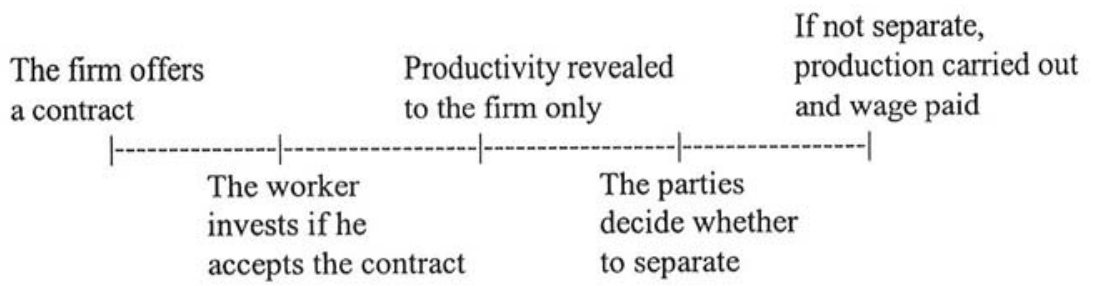

Fig. 1.-Events and their sequence

will continue. For the relationship to continue, both sides must agree to do so. If they do, the production will be carried out and the worker paid according to the contract. Otherwise, there will be no production in this firm. The worker will leave to work for and receive his market wage w from another employer. Figure 1 summarizes these moves and events.

Technology.-The worker's productivity is given by

$$
x=e+\varepsilon,
$$

where $e$ is the worker's SHC investment and $\varepsilon$ a random variable with mean $0 .{ }^{10}$ This specification suggests that the worker's productivity $x$ may take on negative values. This is fine because $x$ is the revenue of the firm net of all but wage cost of the worker.

Denote the probability density function of $\varepsilon$ by $f(y, \sigma)$. Then the probability density function of $x$ is $f(x-e, \sigma) ; \sigma$ is a measure of ULP. An example of $\sigma$ is the standard deviation of $\varepsilon$. We make the following technical assumption:

TA1:

i) For each $\sigma, f(y, \sigma)$ is smooth, unimodal, and there exist $y_{1}(\sigma)<0$ and $y_{2}(\sigma)>0$ such that $f_{\sigma}(y, \sigma)<0$ for $y_{1}(\sigma)<y<y_{2}(\sigma)$ and $f_{o}(y, \sigma)>0$ for $y<y_{1}(\sigma)$ or $y>y_{2}(\sigma)$, where $f_{\sigma}$ represents the partial derivative of $f$ with respect to $\sigma .^{11}$

ii) As $\sigma \rightarrow 0, f(y, \sigma) \rightarrow 0$ for $y \neq 0, f(0, \sigma) \rightarrow \infty, y_{1}(\sigma) \rightarrow 0$, and $y_{2}(\sigma) \rightarrow$ 0 .

iii) As $\sigma \rightarrow \infty, f(y, \sigma) \rightarrow 0$ for all $y, y_{1}(\sigma) \rightarrow-\infty$, and $y_{2}(\sigma) \rightarrow \infty$.

${ }^{10}$ One might ask if it is possible to have $w>0$ but $E(x)=0$ when $e=0$, i.e., the worker has a positive market value but no expected value at the firm if no SHC investment is made. An affirmative answer to the question can be based on the plausible assumption that the worker has positive productivity we doing simple work, say at a gas station, but not in a complex production system without some learning first.

${ }^{11}$ We can weaken the assumption by replacing $f_{\sigma}(y, \sigma)>0$ for all $y<y_{1}(\sigma)$ or $y>y_{2}(\sigma)$ with $f_{\sigma}(y, \sigma) \geq 0$ for all $y<y_{1}(\sigma)$ or $y>y_{2}(\sigma)$ but $f_{\sigma}(y, \sigma)>0$ for $y$ in a left neighborhood of $y_{1}(\sigma)$ or in a right neighborhood of $y_{2}(\sigma)$. The weakened assumption allows us to consider probability distributions with finite support. 
iv) $y_{1}^{\prime}(\sigma) \leq 0$ and $y_{2}^{\prime}(\sigma) \geq 0$.

Technical assumption 1.i implies that, as $\sigma$ increases, some probability density of $\varepsilon$ is spread from the neighborhood of the mean of the distribution to its two tails; in other words, $f\left(y, \sigma_{1}\right)$ dominates $f\left(y, \sigma_{2}\right)$ in the sense of second-order stochastic dominance for $\sigma_{1}<\sigma_{2}$. Note that, in the family of unimodal probability density functions, a mean-preserving spread of probability density has to take the form specified in TA1.i if we require that the region from which probability density is taken away be an interval. Technical assumption 1.ii implies that, as $\sigma \rightarrow 0$, the probability density of $\varepsilon$ concentrates near the mean value 0 and there is almost no uncertainty about the productivity. Technical assumption 1.iii implies that, as $\sigma \rightarrow \infty$, the probability density of $\varepsilon$ is almost evenly spread over $(-\infty, \infty)$ and the level of uncertainty is as high as possible. Technical assumption 1.iv says that the range around the mean of $\varepsilon$ from which probability density is taken away as $\sigma$ increases, $\left(y_{1}(\sigma), y_{2}(\sigma)\right)$, expands (weakly) as $\sigma$ increases; it is only used in the proof of proposition 4. Assumption TA1 is satisfied by, among others, normal, $t$ - and beta distributions. ${ }^{12}$ In each of these families of probability distributions, secondorder stochastic dominance can be parameterized by the standard deviation.

The cost of investment in SHC is $c(e)$, which is assumed to have the following properties:

TA2:

i) $c(0)=c^{\prime}(0)=c^{\prime \prime}(0)=0 ; c^{[m]}(0) \neq 0$ for some $m>2 ; c^{\prime}(e), c^{\prime \prime}(e)$, $c^{\prime \prime \prime}(e)>0$ for all $e>0$; $\lim _{e \rightarrow \infty} c^{\prime}(e)=\infty$.

ii) $c^{\prime \prime \prime}(e) / c^{\prime \prime}(e)<c^{\prime \prime \prime}(e) / c^{\prime}(e)$.

Most of the specifications in TA2 are conventional. The assumption $c^{[m]}(0) \neq 0$ for some $m>2$ is needed to ensure the existence of the limit of $c^{\prime}(e) / c^{\prime \prime}(e)$ as $e$ approaches zero. The assumption $c^{\prime \prime \prime}(e)>0$ is needed to satisfy the second-order condition of the firm's optimization problem. ${ }^{13}$ Part ii of TA2 says that the convexity of $c(e)$ is greater than the convexity of $c^{\prime}(e)$ for all $e \in(0, \infty)$. This assumption is made to keep the problem tractable. ${ }^{14}$ All assumptions in TA2 are satisfied if, for example, $c(e)$ is a power function with the power greater than 2 .

${ }^{12}$ Since the mean of beta distribution $B(p, q)$ is $p /(p+q) \neq 0$, we would need to assume that $\varepsilon / \sigma+p /(p+q)$ follows beta distribution $B(p, q)$.

${ }^{13}$ The proof of this statement is ayailable upon request.

${ }^{14}$ Note that ii can also be written in the form of $c^{\prime}(e) c^{\prime \prime \prime}(e) /\left[c^{\prime \prime}(e)\right]^{2}<1$. Let $h(e)=c^{\prime}(e) / c^{\prime \prime}(e) \cdot h^{\prime}(e)=1-c^{\prime}(e) c^{\prime \prime \prime}(e) /\left[c^{\prime \prime}(e)\right]^{2}$. Assumption ii is not a very strong assumption since the opposite cannot hold for all $e$. If $c^{\prime}(e) c^{\prime \prime \prime}(e) /\left[c^{\prime \prime}(e)\right]^{2}>1$, $h^{\prime}(e)<0$. Assumption $i$ and L'Hopital's rule imply that $\lim _{e \rightarrow 0} h(e)=0$. So $h(e)<0$ for $e>0$. This contradicts the assumptions $c^{\prime}(e)>0$ and $c^{\prime \prime}(e)>0$. 
The two-sided asymmetric information gives the potential for two types of moral hazard problems. First, the worker may choose a low effort level in SHC investment if wage is not closely tied to effort, which is a typical moral hazard problem in agency. However, here an outcome-based incentive cannot provide the worker proper incentives because, with output level as her private information, the employer has a tendency to declare a low output so that she can deny the worker the return to his investment, which is the second type of moral hazard problem. Kahn and Huberman (1988) show that a fixed-wage, up-or-out contract can solve this twosided moral hazard problem. ${ }^{16}$ In such a contract, wage is fixed and above the worker's best alternative in the market. The employment relationship will be terminated if realized productivity is below the fixed wage, or otherwise continue. The contract provides the worker incentives to invest in SHC because it improves the odds of continuing the relationship, which he desires because of the higher wage guaranteed by the contract. At the same time, when realized productivity is above the wage, it is in the employer's self-interest to retain the worker. In the model, we will only consider the case $w>\underline{w}$ for if $w<\underline{w}$ the worker will not accept the contract and if $w=w$ the worker will not invest in SHC even if he accepts the contract. Note that $w>$ w means that the worker never initiates separation.

Under the rule that the relationship is terminated when $x<w$, a wage not be able to do so in a credible way, because the employees cannot effectively interpret the information without sustained experience with the firm's books and participation in its management (Hall and Lazear 1984; Kahn and Huberman 1988; Ben-Ner and Jun 1996). One other possibility is that measures of $x$ are mostly the employer's subjective judgment. See Baker, Gibbons and Murphy (1994) and Prendergast and Topel (1996) for discussions of subjective vs. objective measures of a worker's performance.

${ }^{16}$ We do not consider the issues of revealing information by using mixed strategies or contract renegotiation under asymmetric information. 


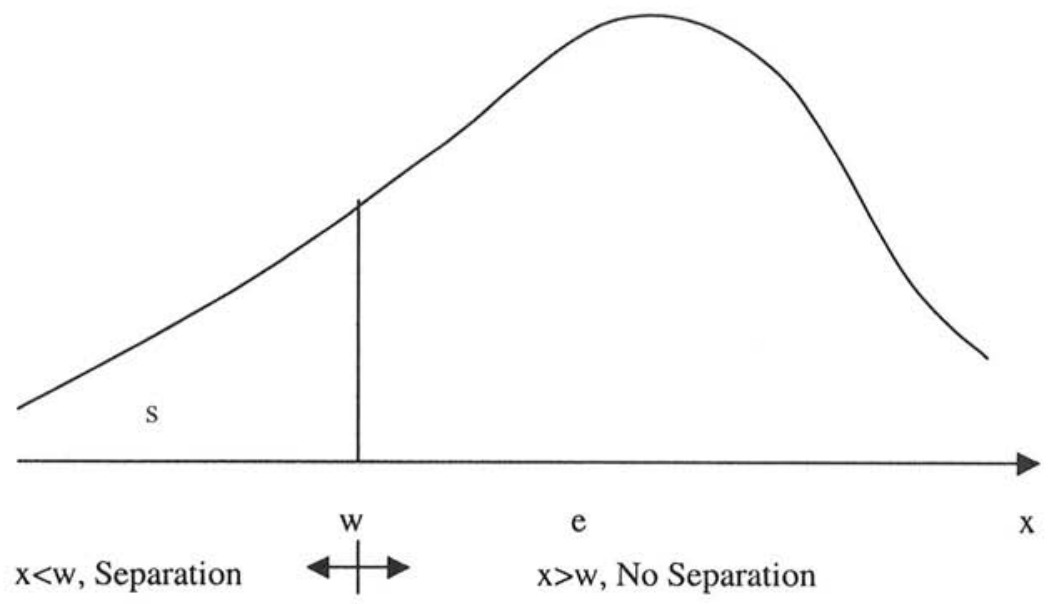

FIG. 2.-The probability density function of $x$

offer $w$ in the contract implies a probability of separation (after SHC investment) given by

$$
s=\operatorname{Pr}\{x<w\}=\int_{-\infty}^{w} f(x-e, \sigma) d x .
$$

The separation rule and its implication on the probability of separation is illustrated by figure 2, in which $e$ is the mean, $w$ the cutoff point, and $s$ the area under the density function and to the left of $w$. When $\varepsilon<$ $w-e$, separation occurs. When $\varepsilon \geqslant w-e$, there is no separation. Note that the density function is not necessarily symmetric on the two sides of $e$.

The players' objectives.-The worker is a risk-neutral, expected net income maximizer whose problem is to

$$
\max _{e} U=s \underline{w}+(1-s) w-c(e),
$$

where $s$ is the probability of separation (given above) and a function of $e$. The risk neutrality assumption will help us to avoid many complications not essential to our analysis.

The firm is also risk-neutral and its objective is to maximize expected 
profit,

$$
\begin{aligned}
& \max _{w} \pi=\int_{w}^{\infty}(x-w) f(x-e, \sigma) d x \\
& \text { subject to: } \begin{array}{l}
\text { (IC) } e=\arg \max U, \\
\text { (IR) } \max U \geq w .
\end{array}
\end{aligned}
$$

The first constraint in the firm's optimization problem is the incentive compatibility constraint and the second the worker's individual rationality constraint.

The final technical assumption we make is:

TA3:

The first-order condition approach is valid for solving the firm's optimization problem.

\section{First-Order and Equilibrium Conditions}

Given the wage offer $w$, the first-order condition of the worker's utility maximization problem is:

$$
\text { FOC-W: } \frac{\partial U}{\partial e}=(w-\underline{w}) \frac{\partial s}{\partial e}-c^{\prime}(e)=(w-\underline{w}) f(w-e, \sigma)-c^{\prime}(e)=0 .
$$

We assume that the second-order condition $\left(\delta^{2} U / \delta e^{2}\right)<0$ is satisfied. Then FOC-W implicitly defines $e$ as a function of $w$ and $\sigma$.

In the firm's optimization problem, constraint IR is automatically satisfied if $w \geq \underline{w}$, since the worker has the option of choosing $e=0$. Constraint IC is replaced by the worker's first-order condition (FOC-W). We then substitute the implicit function defined by FOC-W into the firm's objective function $\pi$. By a change of variable $y=x-e, \pi$ can be rewritten as

$$
\pi=\int_{w-e}^{\infty}(y+e-w) f(y, \sigma) d y
$$

Differentiating the above equation with respect to $w$, we get the firstorder condition of the firm's optimization problem:

$$
\frac{\partial \pi}{\partial w}=\left(\frac{\partial e}{\partial w}-1\right) \int_{w-e}^{\infty} f(y, \sigma) d y=\left(\frac{\partial e}{\partial w}-1\right)(1-s)=0 .
$$

Since $s<1$ in every employment relationship, the above equation is equiv- 
alent to

$$
\frac{\partial e}{\partial w}=1
$$

where $e$ is implicitly defined by FOC-W. This result is very intuitive. For profit maximization, the employer will increase the wage offer by a dollar if it can increase the expected productivity (increase SHC investment) by more than a dollar. She will continue to do so until the last dollar increases expected productivity by exactly one dollar.

By the Implicit Function Theorem and also by FOC-W, $\delta e / \delta w=$ $-\left(\delta^{2} U / \delta e \delta w\right) /\left(\delta^{2} U / \delta e^{2}\right)$. Then, the firm's first-order condition $\delta e / \delta w=$ 1 becomes

$$
\frac{\partial^{2} U}{\partial e \partial w}+\frac{\partial^{2} U}{\partial e^{2}}=0 .
$$

Differentiation of FOC-W yields

$$
\begin{gathered}
\frac{\partial^{2} U}{\partial e \partial w}=f(w-e, \sigma)+(w-\underline{w}) f_{y}(w-e, \sigma), \\
\frac{\partial^{2} U}{\partial e^{2}}=-(w-\underline{w}) f_{y}(w-e, \sigma)-c^{\prime \prime}(e),
\end{gathered}
$$

where $f_{\mathrm{y}}$ represents the partial derivative of $f$ with respect to its first argument. Substitution of (2) and (3) into (1) and simplification yield

$$
\text { FOC-F: } f(w-e, \sigma)-c^{\prime \prime}(e)=0 \text {. }
$$

As we stated earlier, the second-order condition of the firm's optimization problem is implied by TA2. Then the equilibrium is determined by two first-order conditions, FOC-W and FOC-F, which can be combined to obtain

$$
\mathrm{EQ}: h(e)=w-\underline{w},
$$

where $h(e) \equiv c^{\prime}(e) / c^{\prime \prime}(e)$. The equation EQ describes the relationship between $e$ and $w$ in equilibrium, both of them functions of the three parameters: $\underline{w}, \sigma$, and the cost function $c(e)$. From TA2, straightforward computation shows that $h^{\prime}(e) \in(0,1)$. Then EQ implies the following proposition.

Proposition 1. (i) $e$ and $w$ move in the same direction as $\sigma$ changes. (ii) $e$ changes faster than $w$ as $\sigma$ changes.

Note that this result does not depend on assumption TA1. This means that $\sigma$ does not have to be a measure of uncertainty; it could be any parameter related to the probability distribution of $\varepsilon$. This strong result 
is nontrivial. A higher wage makes continuing the employment relationship more attractive to the worker, which conceivably gives the worker more incentive to invest in SHC. However, when wage is too high, it will be very difficult for the worker to avoid being fired by investing in SHC, and thus investment incentive is low. Our analysis suggests that, in equilibrium, wage will not be too high and $e$ is larger when $w$ is larger. The intuition for part ii of the proposition comes from the result $\delta e / \delta w=1$, which we explained earlier. By the chain rule, $d e / d \sigma=$ $(\delta e / \delta w)(d w / d \sigma)+\delta e / \delta \sigma$. Then $\delta e / \delta w=1$ implies that $d e / d \sigma=d w / d \sigma+$ $\delta e / \delta \sigma$. Suppose that $d e / d \sigma$ and $d w / d \sigma$ are both positive. Equilibrium $e$ increases with $\sigma$ only when a higher $\sigma$ improves the worker's incentive to invest for any given contract, that is, $\delta e / \delta \sigma>0$. Therefore $d e / d \sigma=$ $(d w / d \sigma)+\delta e / \delta \sigma>d w / d \sigma$. A similar argument is also valid for the case in which $d e / d \sigma$ and $d w / d \sigma$ are both negative.

\section{Impact of ULP on Wage, SHC Investment, and Separation}

In the previous section, the equilibrium wage offer and SHC investment under the fixed wage contract were characterized as functions of $\underline{w}, c(e)$, and $\sigma$. In this section, we study the comparative statics of the firm's wage offer, the worker's SHC investment, and the probability of separation when there is a change in ULP.

We first consider the effects of ULP on the equilibrium wage offer and on the SHC investment. For this purpose, it is helpful to note that

$$
\operatorname{Sign} \frac{d e}{d \sigma}=\operatorname{Sign} \frac{d w}{d \sigma}=\operatorname{Sign} \frac{\partial e}{\partial \sigma}=\operatorname{Sign}_{\sigma}(w-e, \sigma) .
$$

Lemma 1 in the appendix gives these relationships. To understand the intuition of how investment $e$, wage $w$, and distribution $f$ change with ULP, refer to figure 3. It is clear from FOC-W that, given the contract wage $w>\underline{w}$, the worker chooses $e$ to reduce the probability of separation. The marginal reduction in the probability of separation by investment is $f(w-e, \sigma)$. If $f_{\sigma}(w-e, \sigma)>0$, an increase in $\sigma$ increases the effectiveness of investment in reducing separation. Therefore, the worker invests more in SHC. As wage becomes more effective in inducing investment, the employer also finds it worthwhile to offer a higher wage. The opposite holds if $f_{\sigma}(w-e, \sigma)<0$. Proposition 2 states that, when ULP is low, the equilibrium wage and level of SHC investment both increase with the level of ULP.

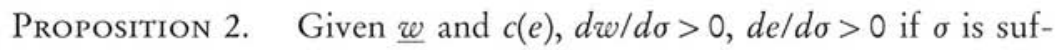
ficiently small.

Proof. First of all, $\lim _{\sigma \rightarrow 0} e \neq \infty$ because otherwise the total expected surplus, $e-c(e)$, will approach negative infinity, and it will then be impossible for the firm to be profitable and the worker to be willing to 


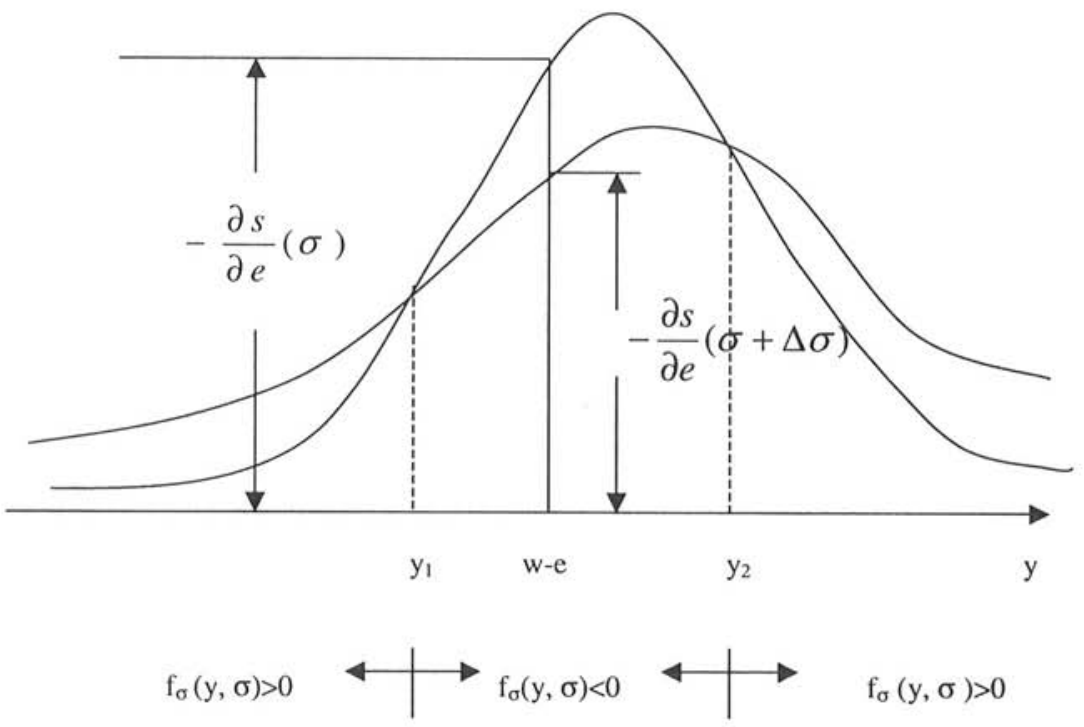

FIG. 3.-Change in the probability density of $\varepsilon$ when $\sigma$ changes

accept the contract. Then, by FOC-F, $\lim _{\sigma \rightarrow 0} f(w-e, \sigma)=\lim _{\sigma \rightarrow 0} c^{\prime \prime}(e)<$ $\infty$. It follows from lemma 2 (in the appendix) that $f(w-e, \sigma)<$ $f\left(y_{i}[\sigma], \sigma\right)$ for $i=1,2$, and sufficiently small $\sigma$. Consequently, $w-e$ cannot be between $y_{1}(\sigma)$ and $y_{2}(\sigma)$, because $f(y, \sigma)$ is unimodal. By the definition of $y_{i}(\sigma)$ in TA1.i, $f_{\sigma}(w-e, \sigma)>0$ for sufficiently small $\sigma$. Our result then follows from lemma 1. Q.E.D.

The intuition for proposition 2 is as follows. When $\sigma$ is very small, the range of $\left(y_{1}(\sigma), y_{2}(\sigma)\right)$ is also very small. At the same time, $f(y, \sigma)$ is very high near the mean of the distribution, that is, around $y=e$. The high $f(y, \sigma)$ near the mean implies that, so long as $e$ is close to $w$, SHC is marginally highly effective in reducing the probability of separation, which is the motivation for the worker to invest in $e$. So more $e$ will be invested, moving the mean of the distribution away from $w$. This will continue until the equilibrium $w$ is outside of the range of $\left(y_{1}(\sigma), y_{2}(\sigma)\right)$. Outside of $\left(y_{1}(\sigma), y_{2}(\sigma)\right), f(y, \sigma)$ increases when $\sigma$ is larger, meaning that, at the original $w, e$ is marginally more effective in reducing the probability of separation. This leads to a new, higher wage offer and increased $e$ at the new equilibrium.

The next proposition is about the case in which the level of ULP is high. It states that, in this case, the equilibrium wage and level of SHC investment both decrease with the level of ULP, which is exactly the opposite of proposition 2 . 
Proposition 3. Given $\underline{w}$ and $c(e), d w / d \sigma<0$ and $d e / d \sigma<0$ if $\sigma$ is sufficiently large.

Proof. By FOC-F, $c^{\prime \prime}(e)=f(w-e, \sigma)$. As $\sigma \rightarrow \infty, f(w-e, \sigma) \rightarrow 0$ by TA1.ii and therefore $e \rightarrow 0$. Then, by L'Hopital's rule and TA2.i, $\lim _{\sigma \rightarrow \infty} c^{\prime}(e) / c^{\prime \prime}(e)=0$. It follows from EQ that $w \rightarrow \underline{w}$. Then, $y_{1}(\sigma)<$ $w-e<y_{2}(\sigma)$, or $f_{o}(w-e, \sigma)<0$ for sufficiently large $\sigma$. By lemma 1 , this implies that $d w / d \sigma<0$ and $d e / d \sigma<0$ for sufficiently large $\sigma$. Q.E.D.

The main reason for the result of proposition 3 is that when $\sigma$ is very large, the range $\left(y_{1}(\sigma), y_{2}(\sigma)\right)$ is also very large (by TA1.iii). But both $w$ and $e$ are bounded so that $(w-e)$ falls within the range given by $\left(y_{1}(\sigma)\right.$, $\left.y_{2}(\sigma)\right)$, which by definition means $f_{o}(w-e, \sigma)<0$ (TA1.i). The inequality $f_{o}(w-e, \sigma)<0$ means that ULP reduces marginal effectiveness of equilibrium $w$ in inducing SHC investment, hence the comparative static result of lower $w$ and $e$ as $\sigma$ increases.

Proposition 2 says that $d e / d \sigma>0$ for small $\sigma$, and proposition 3 says that $d e / d \sigma<0$ for large $\sigma$. Together, they imply that the sign of $d e / d \sigma$ changes at least once. A natural question is, Does the sign of $d e / d \sigma$ change only once? The answer is yes.

Proposition 4. There exists some $\sigma_{1}>0$, such that $d w / d \sigma>0$ and $d e / d \sigma>0$ for $\sigma<\sigma_{1}$, and $d w / d \sigma<0$ and $d e / d \sigma<0$ for $\sigma>\sigma_{1}$.

Proof. Suppose that the sign of $d e / d \sigma$ changes more than once. Then there exists some $\sigma^{\prime}$ such that $d e / d \sigma<0$ in the left neighborhood of $\sigma^{\prime}$ and $d e / d \sigma>0$ in the right neighborhood. We will show that this is impossible, implying the result of the proposition.

At $\sigma^{\prime}, d e / d \sigma=0$. Then by lemma $1, w\left(\sigma^{\prime}\right)-e\left(\sigma^{\prime}\right)=y_{1}\left(\sigma^{\prime}\right)$ or $y_{2}\left(\sigma^{\prime}\right)$. Suppose $w\left(\sigma^{\prime}\right)-e\left(\sigma^{\prime}\right)=y_{1}\left(\sigma^{\prime}\right)$. For $\quad \sigma<\sigma l, \quad w^{\prime}(\sigma)-e^{\prime}(\sigma)=\left[h^{\prime}(e)-\right.$ 1] $e^{\prime}(\sigma)>0$, because $h^{\prime}(e)<1$. Then $w(\sigma)-e(\sigma)<w\left(\sigma^{\prime}\right)-e\left(\sigma^{\prime}\right)=y_{1}\left(\sigma^{\prime}\right)$. The inequality $y_{1}^{\prime}(\sigma) \leq 0$ implies that $y_{1}\left(\sigma^{\prime}\right) \leq y_{1}(\sigma)$. It follows that $w(\sigma)-e(\sigma)<y_{1}(\sigma)$. Consequently, $f_{o}(w[\sigma]-e[\sigma], \sigma)>0$ and hence $d e / d \sigma>0$ for $\sigma<\sigma^{\prime}$, contradicting the definition of $\sigma^{\prime}$. Similarly, if $w\left(\sigma^{\prime}\right)-e\left(\sigma^{\prime}\right)=y_{2}\left(\sigma^{\prime}\right)$, we can derive contradiction in the right neighborhood of $\sigma^{\prime}$. Q.E.D.

The result in proposition 4 is strong and surprising. Its proof is based on assumption TA1.iv, proposition 1.ii, and lemma 1.

We now consider the effect of ULP on the probability of separation. The following proposition contains an incomplete result about this effect.

Proposition 5. Let $\sigma_{1}$ be as defined in proposition 4. There exists a neighborhood of $\sigma_{1}$ in which $d s / d \sigma>0$.

Proof. A change of variable, $y=x-e$, yields

$$
s=\int_{-\infty}^{w} f(x-e, \sigma) d x=\int_{-\infty}^{w-e} f(y, \sigma) d y .
$$


Differentiate the above equation with respect to $\sigma$. We get

$$
\frac{d s}{d \sigma}=\int_{-\infty}^{w-e} f_{\sigma}(y, \sigma) d y+\frac{d(w-e)}{d \sigma} f(w-e, \sigma) .
$$

At $\sigma_{1}, d(w-e) / d \sigma=0$ and by lemma $1, w\left(\sigma_{1}\right)-e\left(\sigma_{1}\right)=y_{1}\left(\sigma_{1}\right)$ or $y_{2}\left(\sigma_{1}\right)$. For $\sigma<\sigma_{1}, d(w-e) / d \sigma=\left(b^{\prime}[e]-1\right) d e / d \sigma<0$. If $w\left(\sigma_{1}\right)-e\left(\sigma_{1}\right)=y_{2}\left(\sigma_{1}\right)$, then $\lim _{\sigma \rightarrow 0} w(\sigma)-e(\sigma)>y_{2}\left(\sigma_{1}\right)>0$. It follows from TA1.ii that $\lim _{o \rightarrow 0} f(w-e, \sigma)=0$, which, by FOC-F, implies that $\lim _{\sigma \rightarrow 0} e=0$. However, $e=0$ cannot be the expected profit-maximizing solution of the firm when $\sigma$ is small. Therefore, $w\left(\sigma_{1}\right)-e\left(\sigma_{1}\right)=y_{1}\left(\sigma_{1}\right)$. It follows from TA1.i that

$$
\frac{d s}{d \sigma}\left(\sigma^{\prime}\right)=\int_{-\infty}^{y_{1}\left(\sigma_{1}\right)} f_{\sigma}\left(y, \sigma_{1}\right) d y>0 .
$$

Therefore, there exists a neighborhood of $\sigma_{1}$ in which $d s / d \sigma>0$. Q.E.D.

As clear from the second equation in the proof, the sign of $d s / d \sigma$ reflects the balance of two terms representing two effects that a change in $\sigma$ has on $s$. The first term in the equation represents the direct effect with given $w-e$, and the second term the indirect effect through induced changes in $w-e$ itself. For $\sigma<\sigma_{1}, s$ is the area in the left tail (i.e., $w-e<y_{1}(\sigma)$ ) of the probability distribution of $\varepsilon$. As $\sigma$ increases, the tail becomes fatter, and therefore the direct effect is positive, that is, a greater $\sigma$ leads to a higher probability of separation. This effect is still positive when $\sigma$ is larger than but close to $\sigma_{1}$. The direct effect becomes ambiguous as $\sigma$ becomes even larger. The sign of the indirect effect is $\left[b^{\prime}(e)-1\right] d e / d \sigma$; it is negative for $\sigma<\sigma_{1}$ but positive for $\sigma>\sigma_{1}$. The total effect therefore is ambiguous most of the time. However, in the neighborhood of $\sigma_{1}$, the sign of $d s / d \sigma$ is unambiguously positive as the direct effect is positive and the indirect effect is close to zero.

Propositions 4 and 5 imply that, as $\sigma$ changes, the probability of separation $s$ sometimes moves in the same direction with the wage offer $w$ and the SHC investment $e$, but other times it moves in the opposite direction. In particular, in the left neighborhood of $\sigma_{1}, w, e$, and $s$ all increase with $\sigma$, but in the right neighborhood of $\sigma_{1}, w$ and $e$ decrease with $\sigma$ while $s$ increases with $\sigma$. The negative relationship of $e$ with $s$ in the right neighborhood of $\sigma_{1}$, that is, less SHC investment associated with increased likelihood separation, is the familiar prediction of the traditional human capital theory. However, in the left neighborhood of $\sigma_{1}$, a positive relationship of $e$ with $s$ is found, that is, more SHC investment is actually associated with increased likelihood of separation. Such a positive rela- 
tionship is exactly the opposite of the prediction of the traditional human capital theory.

\section{Separation When ULP Is Measured by Standard Deviation}

Proposition 5 tells us that, except in the neighborhood of $\sigma_{1}$, the probability of separation and ULP generally have an ambiguous relationship. To obtain more specific results about the total effect of $\sigma$ on $s$, more structure needs to be imposed on the distribution of $\varepsilon$ with respect to the uncertainty measure $\sigma$, which we do in this section.

We assume that $\sigma$, the measure of ULP, is the standard deviation of $\varepsilon$. For any family of random variables that share the same form of probability distribution but have various levels of standard deviation, their probability density functions can be written in the form of

$$
f(y, \sigma)=\frac{1}{\sigma} g\left(\frac{y}{\sigma}\right)
$$

for some probability density function $g(z)$ and $\sigma \in(0, \infty)$. Differentiation of $f(y, \sigma)$ with respect to $\sigma$ yields

$$
f_{o}(y, \sigma)=-\frac{1}{\sigma^{2}}\left[g(z)+z g^{\prime}(z)\right],
$$

where $z=y / \sigma$, which implies that the sign of $f_{o}(y, \sigma)$ is the opposite of that of $g(z)+z g /(z)$. Assumption TA1 can then be replaced by

TA1':

The function $g$ is unimodal, and there exists $z_{1}<0<z_{2}$ such that $g(z)+z g^{\prime}(z)>0$ for $z \in\left(z_{1}, z_{2}\right)$, and $g(z)+z g^{\prime}(z)<0$ for $z<z_{1}$ or $z>z_{2}$.

The function $y_{i}(\sigma)$ defined in TA.ii is $z_{i} \sigma$ in this case.

At the end of the last section, we noticed that a change in ULP has two effects on separation, one direct and one indirect. In the general case considered there, it is difficult to determine the sign of the net and total effect of ULP on separation except in the neighborhood of $\sigma_{1}$. With $\sigma$ specified as the standard deviation of $\varepsilon$, it becomes possible to compare the effects. In particular, this way of parameterizing uncertainty imposes some relationship between $f_{x}(x, \sigma)$ and $f_{s}(x, \sigma)$. This relationship helps us to sign $d s / d \sigma$ globally.

Propositron 6. Let $\sigma_{1}$ again be defined as in proposition 4. There exists some $\sigma_{2}>\sigma_{1}$ such that $d s / d \sigma>0$ for $\sigma<\sigma_{2}$ and $d s / d \sigma<0$ for $\sigma>$ $\sigma_{2}$.

Proof. By the proof of proposition $5, \lim _{o \rightarrow 0}(w-e)=0$, and by the proof of proposition 2, $\lim _{\sigma \rightarrow 0} e \neq \infty$. Then, EQ implies that $\lim _{\sigma \rightarrow 0} w=\lim _{\sigma \rightarrow 0} e=e_{0}$, where $e_{0}$ satisfies equation $e_{0}-\underline{w}=b\left(e_{0}\right)$ and 
therefore $e_{0}>\underline{w}$. By proposition $4, d e / d \sigma>0$ for $\sigma<\sigma_{1}$. It follows that $e>\underline{w}$ for $\sigma \leq \sigma_{1}$. Since $e\left(\sigma_{1}\right)>\underline{w}$ and $\lim _{\sigma \rightarrow \infty} e=0$, there exists some $\sigma_{2}>\sigma_{1}$ such that $e\left(\sigma_{2}\right)=\underline{w}$. Because $d e / d \sigma<0$ for $\sigma>\sigma_{1}, e>\underline{w}$ for $\sigma<$ $\sigma_{2}$ and $e<\underline{w}$ for $\sigma>\sigma_{2}$. It follows from lemma 3 (in the appendix) that $d s / d \sigma>0$ for $\sigma<\sigma_{2}$ and $d s / d \sigma<0$ for $\sigma>\sigma_{2} .{ }^{17}$ Q.E.D.

The seemingly anti-intuitive part of the proposition is $d s / d \sigma<0$ for $\sigma>\sigma_{2}$. For large $\sigma, e$ is very small and $w$ is close to $\underline{w}$. Then $e<w$. With $e<w$, greater ULP means a better chance of $x$ ending up higher than $w$. This means that the direct effect of $\sigma$ on $s$ is negative. Meanwhile, as $\sigma$ approaches infinity, $e$ and $w$ both approach smoothly to some finite values. This implies that $d e / d \sigma$ and $d w / d \sigma$ are both very small. Consequently, the indirect effect of $\sigma$ on $s$ (through $e$ and $w$ ) is very small and dominated by the direct effect. We thus find the sign of $d s / d \sigma$ negative.

The results of propositions 4 and 6 allow us to discuss the relationship between SHC and separation globally. Together, they imply that, if the initial ULP is either very small or very large, SHC investment and the probability of separation move in the same direction when ULP changes. Only in the medium range of $\sigma$ given by $\sigma_{2}>\sigma>\sigma_{1}$ do we see them move in opposite directions as predicted by the traditional human capital theory. Figure 4 summarizes the relationship of SHC investment and separation as ULP changes.

\section{Discussions of the Model's Empirical Implications}

The most important empirical implication of our model is probably that one should not always expect to find a negative correlation between SHC and labor turnover, that is, more SHC leads to less turnover, as conventionally belicved. Although such a negative relationship does exist within certain parameter ranges, a reverse, positive relationship between SHC and labor turnover can also exist when the parameters have other values. More specifically, the findings of our model suggest that a positive correlation between SHC and the probability of separation is likely to be found if the data used in a study are from firms with very low or very high ULP, while a negative correlation is likely to be found if the data used are from firms with medium levels of ULP. If the data used are from firms of very different levels of ULP, then the theory cannot predict the relationship between SHC and the probability of separation. ${ }^{18}$

It is interesting to note that existing empirical evidence of the relationship between SHC and labor turnover is indeed mixed and inconsistent. The work of Mincer (1988) finds that more training (as a measure

${ }^{17}$ Note that the proof of proposition 6 does not use the specification of $\sigma$ as the standard deviation directly; it only uses it through the results of lemma 3 .

is Although, as Brian McCall noted to us, ex post the relationship between SHC and separation is always signed in any particular study. 


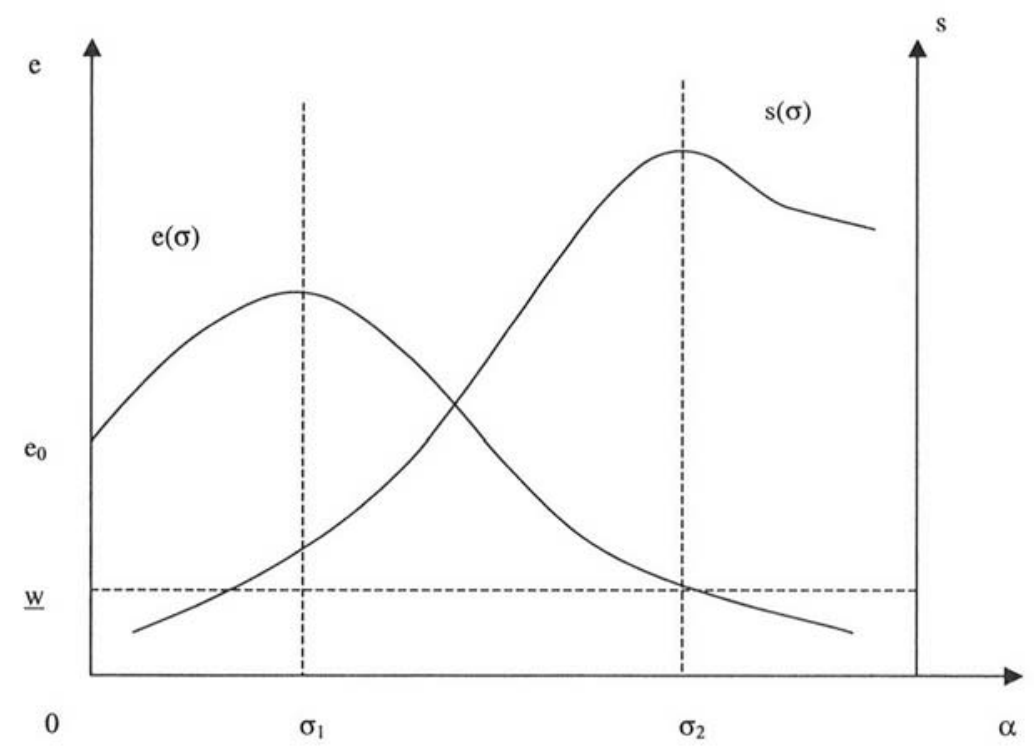

FIG. 4.-Specific human capital investment and the probability of separation at different levels of ULP.

of more SHC investment) lowers separation rates and lengthens job durations. Wholey (1990) finds that tenure depends on formal but not informal training. Working with a data set containing information of over 4,000 employees at more than 80 manufacturing plants in the United States and Japan, Levine (1993) finds that establishments with high levels of training do not have low levels of turnover. Although these empirical studies are not designed with our model in mind and are not direct tests of the model's predictions, the lack of consistent negative empirical relationship between SHC investment and labor turnover is certainly consistent with the predictions of our model. ${ }^{19}$

The results of our model suggest that it is methodologically very important to control ULP when empirically studying the relationship between SHC investment and separation. For this purpose, data should be collected measuring ULP at the firm level, such as variance of labor productivity. ${ }^{20}$ One possible way to control for ULP is to select samples from

${ }^{19}$ Levine (1993) interprets the rcsult as not supporting human capital theory. Our model suggests, however, that lack of evidence of negative corrclations between SHC and turnover cannot be interpreted as evidence refuting human capital theory.

${ }^{20}$ This task could be challenging. For one reason, as our referee noted, productivity distributions are likely to have different shapes in different industries. For another reason, when an up-or-out contract is employed, separation would occur when labor productivity falls below wage. This means truncation of productivity distribution information from below. 
within an occupation or industry with known high or low variances to see in what direction the relationship between SHC and separation moves. ${ }^{21}$ When all the data used in a study are from an occupation or industry in which productivity variations do not span from very low to very high, the study is least likely to need to be concerned with the issue of nonmonotonicity in the relationship between SHC and separation. ${ }^{22}$ When the levels of ULP in a sample span a very large range, it may be worthwhile or even necessary to divide the sample into subsample groups by their levels of ULP to see if the relationship between SHC and separation has different signs across groups, if the signs change across groups according to ULP levels, and if the relationship found in a group is different from that of the whole sample. ${ }^{23}$

\section{Summary and Concluding Remarks}

A fixed-wage model is used to show that a worker's wage and SHC investment can either increase or decrease with uncertainty levels depending on the initial level of ULP. It is well understood and quite intuitive that excessive ULP can discourage SHC investment and thereby harm productivity. Our model confirms such a negative relationship between SHC investment and ULP when the initial level of ULP is high. A new finding of our model, which is not as well understood and not as intuitive, is that too littlc uncertainty can also be detrimental to productivity. When the initial level of ULP is low, an increase in it can actually provide a stronger incentive for the worker to increase SHC investment. This seems to be due to the fact that, when ULP is very low, the worker can easily become complacent in the sense that an SHC investment level slightly above the wage would provide the worker adequate employment security. With the level of employment security already high, the marginal benefit of further SHC investment is low. This result identifies "complacency" as another source of inefficiency in heavily regulated and protected industries, besides the familiar incfficient pricing behavior of firms in these industries. Parallel to the popular proverb in China and Japan that "crisis

${ }^{21}$ We owe thanks to Morris Kleiner for this idea. Kleiner further noticed that investment bankers are known for huge ULP at initial hire, whereas teachers and enginecrs have relatively low ULP to task. He suggested that data of SHC and scparation can be derived from the National Longitudinal Survey or from census data such as Public Use Samples and the Current Population Survey.

${ }^{22}$ We thank Glenn M. MacDonald for the suggestion that the issue of nonmonotonicity may have different importance for studies using different data.

${ }^{23}$ The qualitative nature of our model does not allow us to give quantitative recommendations on critical points in ULP levels by which a sample should be divided. We suspect a trial and error process would be needed in most cases to find these critical points. 
creates opportunity," the result here can be phrased as "more uncertainty creates more room for incentives."

Specific human capital investment and the probability of separation do not generally have a monotonic negative relationship predicted by traditional human capital theory. Assuming that ULP is measured by the variance of productivity, we have shown that SHC and the probability of separation are positively correlated if ULP is either very small or very large, and negatively correlated if ULP is in the medium range. The positive correlation between the probability of separation and SHC investment need not be as counterintuitive as one might think. For example, one way to eliminate complacency in a firm with a very high level of job security is to expose it to more uncertainty and thereby reduce the level of job security. As this happens, higher effort and more separations can be both expected.

A few words are due about why a nonmonotonic relationship between SHC investment and separation is obtained in this work, while previous work obtained a negative relationship. The explanation lies in contract forms. By focusing on how the employer and the worker "split the gain" of SHC investment (and probably also share the cost), previous work implicitly assumed a sharing contract. Under a sharing contract, separation is efficient and occurs when the worker's productivity falls below his market wage. Since in a partial equilibrium model market wage is typically assumed exogenously given, the probability of separation is always reduced when more SHC investment shifts the mean of the productivity distribution further to the right. The result differs in the fixed wage model where the wage offer is endogenous and changes with ULP. These strikingly different results underscore the great importance of explicitly stating the contractual form assumed when studying SHC investment in employment relations.

Our work offers a new, competing explanation of international differences in employment practices. For example, to explain U.S.-Japanese differences in employment stability and human capital investment, Abegglen (1958), Prendergast (1989), and Glacser (1991) introduce the theory of self-enforcing multiple equilibria. Mincer and Higuchi (1988) suggest that Japanese workers invest more in SHC because higher growth rates in Japan make SHC more productive and therefore more valuable. ${ }^{24} \mathrm{Hash}-$ imoto (1990) attributes more SHC investment to lower SHC investment costs in Japan (because of more rigorous general education). Our model suggests that one can also look at the role of ULP. If the parameters are

${ }^{24}$ Chang and Wang (1995), however, show that conditions in productivity distribution and SHC investment cost must be satisficd for multiple equilibria to exist. Glaeser (1992) comments that it is not clear why growth-inspired SHC investment should be more important than growth-inspired matching. 
right, greater ULP in the United States can explain why American workers invest less in SHC and have higher turnover rates than their Japanese counterparts. ${ }^{25}$

While important results have been obtained, more work is called for to study the effect of ULP on employment relations. The effect of ULP on SHC investment is likely to be different when information concerning a worker's realized productivity is symmetric so that wage can be determined through bargaining. The effects of ULP on other important labor market behaviors, such as job search (Mortensen 1978; Jovanovic 1979b) and learning about worker ability (Farber and Gibbons 1996), are also important topics for investigation.

\section{Appendix}

LEMMA 1.

$$
\operatorname{Sign} \frac{d e}{d \sigma}=\operatorname{Sign} \frac{d w}{d \sigma}=\operatorname{Sign} \frac{\partial e}{\partial \sigma}=\operatorname{Sign} f_{\sigma}(w-e, \sigma) .
$$

Proof. The first equality is a restatement of proposition 1.i. To prove the second equality, differentiate EQ with respect to $\sigma$ to obtain

$$
h^{\prime}(e)\left(\frac{\partial e}{\partial w} \frac{d w}{d \sigma}+\frac{\partial e}{\partial \sigma}\right)=\frac{d w}{d \sigma},
$$

which by the fact that $\delta e / \delta w=1$ (from FOC-F), and after rearranging, is

$$
\frac{d w}{d \sigma}\left(\frac{1}{h^{\prime}(e)}-1\right)=\frac{\partial e}{\partial \sigma} .
$$

Since $h^{\prime}(e) \in(0,1)$, the second equality in the lemma follows.

To prove the third equality, use the FOC-W and the Implicit Function Theorem to obtain $\delta e / \delta \sigma=-\left(\delta^{2} U / \delta e \delta \sigma\right) /\left(\delta^{2} U / \delta e^{2}\right)$. Under the assumption that $\delta^{2} U / \delta e^{2}<0, \delta e / \delta \sigma$ and $\delta^{2} U / \delta e \delta \sigma$ have the same sign. Since, by FOC-W,

$$
\frac{\partial^{2} U}{\partial e \partial \sigma}=(w-\underline{w}) f_{\sigma}(w-e, \sigma),
$$

the third equality in the lemma holds because $w>$ w. Q.E.D.

${ }^{25}$ It is, of course, an empirical question if ULP is in the right range in both the United States and Japan for ULP to explain their differences in SHC investment and employment stability. In n. 2 above, we mentioned evidence of greater ULP in the United States than in Japan in the 1970s and 1980s. The experiences in the 1990 s seem to suggest that Japan has passed the "catch-up" stage and faces greater ULP today than before and also greater difficulty in maintaining their current employment practices. 
Lemma 2. $\lim _{\sigma \rightarrow 0} f\left[y_{1}(\sigma), \sigma\right]=\lim _{\sigma \rightarrow 0} f\left[y_{2}(\sigma), \sigma\right]=\infty$, where $y_{1}(\sigma)$ and $y_{2}(\sigma)$ are defined in TA1.i.

Proof. Suppose $\lim _{\sigma \rightarrow 0} f\left[y_{1}(\sigma), \sigma\right]=F_{1}<\infty$. Then $f\left[y_{1}(\sigma), \sigma\right]<F_{1}+1$ for sufficiently small $\sigma$. Since $f(0, \sigma)=\infty, f(0, \sigma)>F_{1}+1$ for sufficiently small $\sigma$. Then, there exists some $\tau_{1}(\sigma)$ in the interval $\left(y_{1}(\sigma), \min \{m(\sigma), 0\}\right)$, where $m(\sigma)$ is the mode of $f(y, \sigma)$, such that $f\left[\tau_{1}(\sigma), \sigma\right]=F_{1}+1$. Since $\tau_{1}(\sigma)$ is less than $m(\sigma), f_{y}\left[\tau_{1}(\sigma), \sigma\right]>0$. By TA1.i, $f_{\sigma}\left[\tau_{1}(\sigma), \sigma\right]<0$ because $\tau_{1}(\sigma)$ is between $y_{1}(\sigma)$ and 0 and therefore is between $y_{1}(\sigma)$ and $y_{2}(\sigma)$. By the definition of $\tau_{1}(\sigma)$,

$$
\left.0=\frac{d}{d \sigma}\left\{f\left[\tau_{1}(\sigma), \sigma\right]\right\}=f_{y}\left[\tau_{1}(\sigma), \sigma\right] \tau_{1}^{\prime}(\sigma)+f_{\sigma}\left[\tau_{1}(\sigma), \sigma\right]\right) .
$$

It then follows that $\tau_{1}^{\prime}(\sigma)>0$, which together with $\tau_{1}(\sigma)<0$ implies that $\lim _{\sigma \rightarrow 0} \tau_{1}(\sigma)<0$. Consequently, $\lim _{\sigma \rightarrow 0} f\left[\tau_{1}(\sigma), \sigma\right]=0$, contradicting the definition of $\tau_{1}(\sigma)$. Therefore, $\lim _{\sigma \rightarrow 0} f\left[y_{1}(\sigma), \sigma\right]=\infty$. Similarly, we can prove $\lim _{\sigma \rightarrow 0} f\left[y_{2}(\sigma), \sigma\right]=\infty$. Q.E.D.

LEMma 3. $d s / d \sigma>0$ if in equilibrium $e>\underline{w}$, and the opposite is true if in equilibrium $e<w$.

Proof. A change of variable, $t=(y-e) / \sigma$, yields

$$
s=\int_{-\infty}^{w} \frac{1}{\sigma} g\left(\frac{x-e}{\sigma}\right) d x=\int_{-\infty}^{z} g(t) d t,
$$

where $z=(w-e) / \sigma$. Take the derivative of $s$ with respect to $\sigma$ to obtain

$$
\frac{d s}{d \sigma}=g(z) \frac{d z}{d \sigma},
$$

which implies that $d s / d \sigma$ has the same sign as $d z / d \sigma$. Rearranging terms in FOC-W, we have

$$
\begin{aligned}
\frac{\partial U}{\partial e} & =\frac{w-\underline{w}}{\sigma} g\left(\frac{w-e}{\sigma}\right)-c^{\prime}(e) \\
& =\frac{w-e+e-\underline{w}}{\sigma} g(z)-c^{\prime}(e) \\
& =\left(z+\frac{e-\underline{w}}{\sigma}\right) g(z)-c^{\prime}(e) .
\end{aligned}
$$

Define the right-hand side of the above equation to be $G(z, e, \sigma)$. Differentiate $\partial U / \partial e$ with respect to $e$. Then

$$
\frac{\partial^{2} U}{\partial e^{2}}=-\frac{1}{\sigma} \frac{\partial G}{\partial z}+\frac{\partial G}{\partial e}=-\frac{1}{\sigma} \frac{\partial G}{\partial z},
$$

where $\delta G / \delta e$ drops out because by FOC-F, $\delta G / \delta e=(1 / \sigma) g(z)-$ $c^{\prime \prime}(e)=0$. Then $\delta G / \delta z>0$ because $\delta^{2} U / \delta e^{2}<0$. Differentiating FOC-W 
with respect to $\sigma$ and using $\delta G / \delta e=0$, we obtain $(\delta G / \delta z)(d z / d \sigma)+$ $\delta G / \delta \sigma=0$. Since $\delta G / \delta z>0, d z / d \sigma$ has the same sign as

$$
-\frac{\partial G}{\partial \sigma}=\frac{e-\underline{w}}{\sigma^{2}} g(z),
$$

which is positive if $e>\underline{w}$; and negative if $e<\underline{w}$. Q.E.D.

\section{References}

Abegglen, James C. The Japanese Factory. Glencoe: Frec Press, 1958.

Acemoglu, Daron, and Zilibotti, Fabrizio. "Was Prometheus Unbound by Chance? Risk, Diversification, and Growth." Journal of Political Economy 105 (August 1997): 709-51.

Aoki, Masahiko. "Toward an Economic Model of the Japanese Firm." Journal of Economic Literature 28 (March 1990): 1-27.

Bai, Chong-En, and Wang, Yijiang. "Output Fluctuations in the Manufacturing Industries in the United States and Japan." Working paper. Minneapolis: University of Minnesota, 1996.

Baker, George; Gibbons, Robert S.; and Murphy, Kevin J. "Subjective Performance Measures in Optimal Incentive Contracts." Quarterly Journal of Economics 109 (November 1994): 1125-56.

Becker, Gary S. Human Capital: A Theoretical and Empirical Analysis, with Special Reference to Education. Chicago: University of Chicago Press, 1975.

Ben-Ner, Avner, and Jun, Byoung. "Employee Buyout in a Bargaining Game with Asymmetric Information." American Economic Review 86 (June 1996): 502-23.

Bernhardt, Dan, and Timmis, Gerald C. "Multiperiod Wage Contracts and Productivity Profiles." Journal of Labor Economics 8 (October 1990): 529-63.

Braudel, Fernand. Civilization and Capitalism. Vol. 2, The Wheels of Commerce. New York: Harper \& Row, 1979.

Carmichael, Lorne. "Firm-Specific Human Capital and Promotion Ladders." Bell Journal of Economics 14 (Spring 1983): 251-58.

Carmichael, Lorne, and MacLcod, Bentley W. "Multiskilling, Technical Change and the Japanese Firm." Economic Journal 103 (January 1993): 142-60.

Chang, Chun, and Wang, Yijiang. "A Framework for Understanding Differences in Labor Turnover and Human Capital Investment." Journal of Economic Behavior and Organization 28 (September 1995): 91-105.

___. "Human Capital Investment under Asymmetric Information: The Pigovian Conjecture Revisited." Journal of Labor Economics 14 (July 1996): 505-19.

DeVrics, Jan. The Economy of Europe in an Age of Crisis, 1600-1750. Cambridge: Cambridge University Press, 1990.

Dixit, A., and Pindyck, R. Investment under Uncertainty. Princeton, NJ: Princeton University Press, 1994. 
Farber, Henry S., and Gibbons, Robert. "Learning and Wage Dynamics." Quarterly Journal of Economics 111 (November 1996): 1007-1147.

Gibbons, Robert, and Waldman, Michael. "Careers in Organizations: Theory and Evidence." In Handbook of Labor Economics, vol. 3, edited by O. Ashenfelter and D. Card. Amsterdam: North-Holland, 1999.

Glaeser, Edward. "Reputation and Turnover in the U.S. and Japan." Photocopied. Cambridge, MA: Harvard University, 1991.

-_. "Two Essays on Information and Labor Markets." Ph.D. dissertation, University of Chicago, 1992.

Hall, Robert E., and Lazear, Edward P. "The Excess Sensitivity of Layoffs and Quits to Demand." Journal of Labor Economics 2 (April 1984): 233-57.

Hashimoto, Masanori. "Specific Human Capital as a Shared Investment." American Economic Review 71 (June 1981): 475-81.

. "Employment and Wage Systems in Japan and Their Implications for Productivity." In Paying for Productivity: A Look at the Evidence, edited by Alan Blinder. Washington: Brookings Institution, 1990.

Hashimoto, Masanori, and Yu, Ben T. "Specific Capital, Employment Contracts and Wage Rigidity." Bell Journal of Economics 11 (Autumn 1980): $536-49$.

Hicks, John R. "Annual Survey of Economic Theory: The Theory of Monopoly." Econometrica 3 (January 1935): 1-20.

Horiuchi, Akiyoshi; Packer, Frank; and Fukuda, Shin'ichi. "What Role Has the 'Main Bank' Played in Japan?" Journal of the Japanese and International Economies 2 (June 1988): 159-80.

Johnson, Chalmers. "Market Rationality vs. Plan Rationality." In Inside the Japanese System, edited by Daniel I. Okimoto and Thomas P. Rohlen, pp. 215-20. Stanford, CA: Stanford University Press, 1988.

Jovanovic, Boyan. "Firm-Specific Capital and Turnover." Journal of Political Economy 87 (December 1979): 1246-60. (a)

_- "Job Matching and the Theory of Turnover." Journal of Political Economy 87 (October 1979): 972-90. (b)

Justice, Betty. Unions, Workers, and the Law. Washington, DC: Bureau of National Affairs, 1983.

Kahn, Charles, and Huberman, Gur. "Two-Sided Uncertainty and 'Upor-Out' Contracts." Journal of Labor Economics 6 (October 1988): 423-43.

Kennan, John. "Bonding and the Enforcement of Labor Contracts." Economics Letters 3 (January 1979): 61-66.

Keynes, John Maynard. The General Theory of Employment, Interest, and Money. Cambridge: Macmillan Cambridge University Press, for the Royal Economic Society, 1936.

Kleiner, Morris. "Public Policy Implications of Financial Information Requirements of National Labor Relations Act." Journal of Accounting and Public Policy 3 (Winter 1984): 253-57.

Levine, David. "Worth Waiting For? Delayed Compensation, Training, and Turnover in the United States and Japan." Journal of Labor Economics 11 (October 1993): 724-52. 
Lewin, David. "Opening the Books: Corporate Information-Sharing with Employees." Research Bulletin no. 167. New York: The Conference Board, 1984.

McCall, Brian. "The Effect of Job Heterogeneity on Reservation Wages." International Economic Review 35 (August 1994): 773-91.

McCloskey, Donald. "English Open Fields as Behavior towards Risk." In Research in Economic History 1, edited by P. Uselding, pp. 124-70. Greenwich, CT: JAI Press, 1976.

Miller, Robert A. "Job Matching and Occupational Choice." Journal of Political Economy 92 (December 1984): 1086-1120.

Mincer, Jacob. "On-the-Job Training: Costs, Returns and Some Implications." Journal of Political Economy 70 (October 1962): 50-79.

—_. "Job Training: Costs, Returns and Wage Profiles." Working Paper no. 3208. Cambridge, MA: National Bureau of Economic Research, 1988.

Mincer, Jacob, and Higuchi, Yoshio. "Wage Structures and Labor Turnover in the United States and Japan." Journal of the Japanese and International Economies 2 (June 1988): 97-133.

Mortensen, Dale. "Specific Capital and Labor Turnover." Bell Journal of Economics 9 (Autumn 1978): 572-86.

North, Douglas, and Thomas, Robert. The Rise of the Western World: A New Economic History. Cambridge: Cambridge University Press, 1973.

Oi, Walter. "Labor as a Quasi-Fixed Factor." Journal of Political Economy 70 (December 1962): 538-55.

Parsons, Donald. "Specific Human Capital: An Application to Quit Rates and Layoff Rates." Journal of Political Economy 80 (NovemberDecember 1972): 1120-43.

—. "The Employment Relationship: Job Attachment, Work Effort, and the Nature of Contracts." In Handbook of Labor Economics, vol. 2, edited by O. Ashenfelter and R. Layard, pp. 789-848. Amsterdam: Elsevier Science, 1986.

Prendergast, Canice. "Multiple Equilibria in the United States and Japan." Photocopied. Chicago: University of Chicago, 1989.

- "The Role of Promotion in Inducing Specific Human Capital Acquisition." Quarterly Journal of Economics 108 (May 1993): 523-34.

Prendergast, Canice, and Topel, Robert H. "Favoritism in Organizations." Journal of Political Economy 104 (October 1996): 958-78.

Rosen, Sherwin. "Learning and Experience in the Labor Market." Journal of Human Resources 7 (Summer 1972): 326-42.

Rosenbaum, James E., and Kariya, Takehiko. "From High School to Work: Market and Institutional Mechanisms in Japan." American Journal of Sociology 94 (May 1989): 1334-65.

Scoones, David. "Matching and Competition for Human Capital," Labour Economics 7 (March 2000): 135-52.

Scoones, David, and Bernhardt, Dan. "Promotion, Turnover and Human Capital Acquisition." Journal of Labor Economics 16 (January 1998): $122-41$.

Shapiro, Carl, and Stiglitz, Joseph E. "Equilibrium Unemployment as a 
Worker Discipline Device." In Efficiency Wage Models of the Labor Market, edited by George A. Akerlof and Janet L. Yellen, pp. 45-56. Cambridge: Cambridge University Press, 1986.

Taylor, John B. "Differences in Economic Fluctuations in Japan and the United States: The Role of Nominal Rigidities." Journal of the Japanese and International Economies 3 (June 1989): 127-44.

Waldman, Michael. "Up-or-Out Contracts: A Signaling Perspective." Journal of Labor Economics 8 (April 1990): 230-50.

Wholey, Douglas. "The Effects of Formal and Informal Training on Tenure and Mobility in Manufacturing Firms." Sociological Quarterly 31 (Winter 1990): 37-57. 